\title{
Optical Emission Analysis of Slag Surface Conditions and Furnace Atmosphere during Different Process Stages in Electric Arc Furnace (EAF)
}

\author{
Matti AULA, ${ }^{1,3) *}$ Ari MÄKINEN, ${ }^{2)}$ Ahti LEPPÄNEN, ${ }^{3)}$ Marko HUTTULA $^{4)}$ and Timo FABRITIUS ${ }^{1)}$ \\ 1) Process Metallurgy Research Group, P.O. Box 4300, Fl-90014, University of Oulu, Finland. \\ 2) Optoelectronics and Measurement Techniques Laboratory, P.O. Box 4500, FI-90014, University of Oulu, Finland. \\ 3) Luxmet Ltd, Paavo Havaksen tie 5 D, Fl-90570, Oulu, Finland. \\ 4) Electron Spectroscopy Group, P.O. Box 3000, FI-90014, University of Oulu, Finland.
}

(Received on February 10, 2015; accepted on April 15, 2015)

\begin{abstract}
In electric arc furnace (EAF) steelmaking the control of the overall process has traditionally been in the hands of the operator. The current level of automation has not allowed for full dynamic control of EAF. In this study new information was sought with a recently tested method of on-line in situ optical emission spectroscopy. Optical emission spectra were measured from Outokumpu Stainless Oy, Tornio works, electric arc furnace 2 during the course of one month. The focus of the study was the analysis of slag surface conditions and alkali emission lines during different process stages. The results show that alkali excitation lines are different in different process periods. According to the properties and appearance the optical emission of alkali metals, in early periods of EAF process the alkali transitions are caused by volatilization of contaminants in the EAF charge. In the later periods the optical emissions of alkali metals are linked to occasional high gas generation, which is likely caused by oxidation-reduction reactions occurring inside the melt. The thermal radiation of the heat was analyzed during different process periods. According to the scrap melting indicator calculated from the measured spectra, the melting conditions vary significantly between heats. The scrap melting indicators have a correlation to the metal yield, but it is obstructed by many different factors affecting the metal yield. The results indicate that on-line in situ optical emission spectrometry can be used in the analysis of scrap melting and slag surface conditions in EAF steelmaking.
\end{abstract}

KEY WORDS: electric arc furnace; optical emission; spectrometry; in situ measurement; stainless steelmaking.

\section{Introduction}

In electric arc furnace (EAF) steelmaking the control of the overall process has traditionally been in the hands of the operator. The current level of automation has not allowed for full dynamic control of EAF. Recently new methods for automatic control of some sub processes have been developed, such as for the blowing of carbon and oxygen ${ }^{1)}$ and tapping the furnace. ${ }^{2)}$

The most important factor holding back the dynamic automation of important process parameters is the lack of information about the process conditions inside the EAF. Currently there are very few methods for gaining information on scrap melting, as well as foaming and decarburization inside EAFs. Continuous on-line measurements are limited to water panel temperatures, arc noise ${ }^{3)}$ and off-gas properties such as composition, ${ }^{4)}$ temperature and flow rates. ${ }^{5)}$ In addition to these measurements, discrete information on melt compositions and temperatures are obtained by manual or robotic probes. Among the oldest control systems

\footnotetext{
* Corresponding author: E-mail: matti.aula@oulu.fi

DOI: http://dx.doi.org/10.2355/isijinternational.ISIJINT-2015-042
}

in EAF automation applications are the automatic control of electrode arms and the control of voltage flicker. ${ }^{6,7)}$ Common to these continuous on-line measurements are that the measured quantities are obtained from outside the EAF and they are indirectly related to the phenomena analyzed. The consequence is that they provide accurate information only for very specific applications, such as the control of slag foaming or carbon injection.

This study seeks to find new means of determining online information about process conditions by conducting optical emission spectrum measurements of the EAF. Previous measurements have focused on the characteristics of optical emission spectra obtained from the industrial EAF. ${ }^{8}$ ) This study focuses on the spectrum types related to the thermal radiation of hot slag or gas and excitation of alkali metals by the exothermic reactions inside the EAF. The advantage of analyzing these types of optical emissions is that they are very frequently observed in industrial furnaces and do not rely on exactly correct aiming of optical fiber, which is required to gain statistically relevant information on the optical emission spectrum of an electric arc.

The optical emission spectrum observed from the EAF depends heavily on the fuming conditions. The measure- 
ments of particle emissivity of BOF off-gas suggests that fumes generated during decarburization of steel are optically thick. ${ }^{9)}$ On the other hand, when gas generation in the EAF is low, the EAF atmosphere may be optically thin ${ }^{8)}$ making it possible to measure slag surface conditions.

\section{Experimental Methods}

The equipment for the analysis of an electric arc emission spectrum consists of a measurement head, optical fiber, fiber protection sleeve and spectrometer. The system for emission spectrum measurement is presented in Fig. 1.

An Ocean Optics $600 \mu \mathrm{m}$ fiber was used in the measurements. The measurement head was a steel pipe holding the end of the optical fiber inside. A hole in the end of the measurement head acted as an aperture for the measurement cone. The measurement cone was defined by the diameter of the hole in the measurement head and the distance between the hole and the optical fiber. The hole used in the measurements was of $5 \mathrm{~mm}$ diameter and the distance to the optical fiber was $15 \mathrm{~cm}$. This configuration produced an optical fiber viewing spot of $15 \mathrm{~cm}$ diameter on the slag surface. The measurement head was installed into an unused lime injection collar on the EAF roof. Similar positioning was used successfully in the previous measurements of an EAF optical emission spectrum. ${ }^{8)}$

An AvaSpec-ULS3648 High-resolution Spectrometer was used in the measurements. The grating of the spectrometer was $600 \mathrm{l} / \mathrm{mm}$ with $500 \mathrm{~nm}$ blaze, and the width of the entry slit was $10 \mu \mathrm{m}$. The wavelength range of the spectrometer was 300-800 $\mathrm{nm}$. The optical resolution of the spectrometer configuration is $0.3 \mathrm{~nm}$, which is sufficient for analysis of alkali emission lines. The measurements were conducted

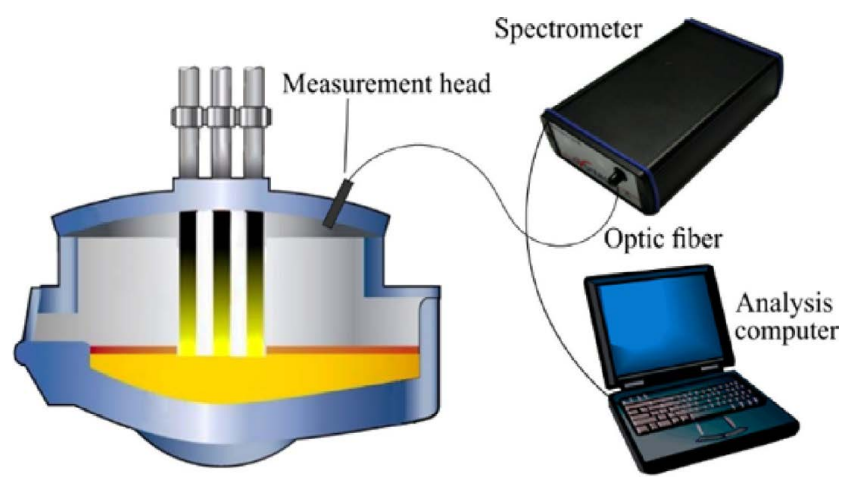

Fig. 1. Schematic view of the experimental setup. ${ }^{10)}$ (Online version in color.) with a 150 millisecond integration time and an average of 8 frames, which results in single spectrum frame representing a time period of 1.2 seconds. CCD averaging was used to reduce dark noise, which was considerable due to the lack of CCD cooling.

Emission spectra were measured from a $140 \mathrm{t}$ AC EAF at Outokumpu Stainless Oy, Tornio Works. The production line in question mainly produces austenitic stainless steels. In total, 70 heats were analyzed during the one month measurement period. The measurement system was installed into the furnace roof and continuously gathered data during the entire measurement period. The only problem during the measurement period was the accumulation of slag on the lime collar. The accumulated slag created an arch on the lime collar and blocked the view to the furnace, which could only be removed in weekly maintenance.

During the measurement period no carbon or oxygen was injected to the furnace. This minimized the dust and $\mathrm{CO}$ generation in the furnace atmosphere and kept the furnace atmosphere as optically thin as possible.

\subsection{Emission Spectrum Analysis}

The data obtained from the measurements was processed with in-house spectral analysis software. The analysis software makes use of a prebuilt ROOT library. ${ }^{11)}$ The data was analyzed by calculating spectrum indicators which describe the most important spectrum features, namely the average temperature of the viewing cone and intensity of alkali emission lines. Different indicators and their descriptions are presented in Table $\mathbf{1 .}$

In-house spectral analysis software was also used in the correction of distortions caused by the optical fiber, the grating and the CCD of the spectrometer, as well as in removal of dark current. The dark current was measured at the start of the measurement period and the dark current frame was removed from all the subsequent frames. Static dark current removal was found to reduce the level of dark current to approximately \pm 30 counts.

The average temperature of the viewing cone was based on the assumption of the material in viewing cone being a gray body. The temperature of the emitting material was calculated according to Eq. (1), which is derived from the ratio of the intensities of different wavelengths according to Wien's law. The average temperature was calculated by relative differences in average intensities of $600 \mathrm{~nm}$ and $800 \mathrm{~nm}$.

$$
T=\left(\frac{1}{\lambda_{2}}-\frac{1}{\lambda_{1}}\right) \frac{h c / k}{\ln \left[R\left(\lambda_{1} / \lambda_{2}\right)^{5}\right]}
$$

Table 1. Indicators calculated from the spectrum and their descriptions.

\begin{tabular}{lll}
\hline \multicolumn{1}{c}{ Indicator name } & Description & Related process phenomena \\
\hline Two-point temperature & $\begin{array}{l}\text { Temperature of emitting material calculated from relative intensities of } \\
600 \text { and } 800 \mathrm{~nm}\end{array}$ & $\begin{array}{l}\text { Average temperature of the material in the } \\
\text { measuring area }\end{array}$ \\
$\mathrm{Na} \mathrm{I} 589 \mathrm{~nm}$ intensity & Area of Voigtian peak fitted to a $589 \mathrm{~nm}$ region & Excitation conditions in the measuring area \\
$\mathrm{K} \mathrm{I} 769 \mathrm{~nm}$ intensity & Area of Voigtian peak fitted to a $769 \mathrm{~nm}$ region & Excitation conditions in the measuring area \\
$\mathrm{Na} \mathrm{I} 589 \mathrm{~nm}$ FWHM & The width of the peak calculated at half of the maximum peak intensity & Magnitude of the line broadening \\
$\begin{array}{l}\text { Na I } 589 \mathrm{~nm} \text { absorption } \\
\text { coefficient }\end{array}$ & Area of negative Voigtian peak fitted to a $589 \mathrm{~nm}$ area & $\begin{array}{l}\text { Absorption by sodium containing fumes } \\
\text { and dusts in the EAF atmosphere }\end{array}$ \\
\hline
\end{tabular}


Where $\lambda_{2}$ is the wavelength of the second measured intensity band, $\lambda_{1}$ is the wavelength of the first measured intensity band, $h$ is the Planck constant, $c$ is the speed of light, $k$ is the Boltzmann constant and $\mathrm{R}$ is the intensity ratio.

The temperature calculated from the intensities of two wavelengths provides a fast tool for analyzing relative differences in spectrum temperature. However, the absolute accuracy of the calculated temperatures relies on the accuracy of the assumptions used in the temperature calculation, namely the gray body assumption, fiber transmission, grating transmission and CCD quantum efficiency. While the optical characteristics of the measurement system are documented by their suppliers, the accuracy of gray body assumption is difficult to measure because it would require prior information about the optical characteristics of the slag surface and EAF atmosphere. Because of these uncertainties, the emphasis of the temperature analysis is on the relative changes of temperature and not the absolute values of the calculated temperatures.

The second and third indicators in Table 1 describe the intensity of the atomic sodium and potassium excitation lines. The exothermic oxidation reactions generate heat, which causes excitation of alkali metal particles inside the flames. The excitation lines Na I 588.995 nm, Na I 589.592 $\mathrm{nm}$ and K I $766.490 \mathrm{~nm}$, K I $769.896 \mathrm{~nm}$ were approximated with Voigtian functions and the intensity of the excitation lines was measured by calculating areas of the Voigtian curves. The width of the Na I $589 \mathrm{~nm}$ emission line is described by calculating the full width of the peak at half the intensity of the peak maximum (FWHM).

The shape of alkali emission line can be affected by absorption, which needs to be accounted to enable comparison of alkali peaks characteristics. In this work the degree of absorption is defined by fitting a negative Gaussian curve in the middle of the $589 \mathrm{~nm}$ region. This method creates some errors in the calculation of absolute line intensities, but provides a fast tool for analyzing relative line shapes.

\section{Results and Discussion}

The results and analysis are divided into different EAF processing periods since the interpretation of the observed emission spectrum is very much related to the stage of the EAF process. These stages are illustrated in Fig. 2 along

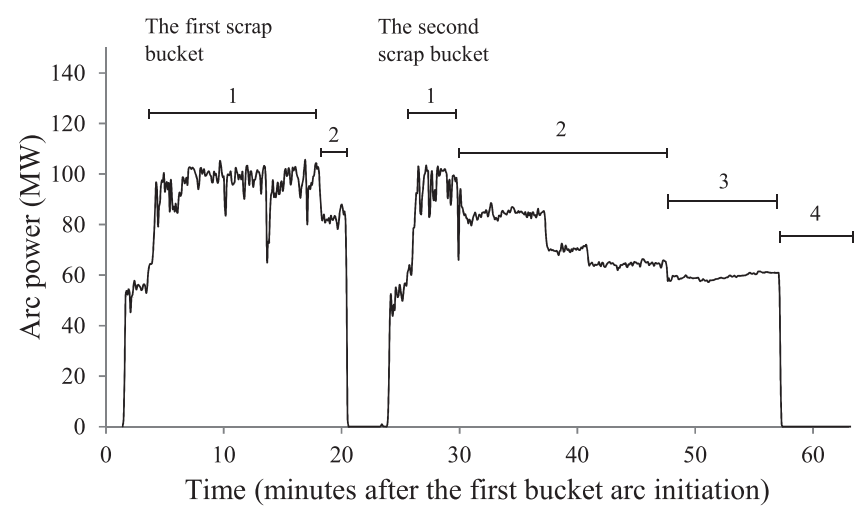

Fig. 2. Definition of different EAF process periods based on arc power (1. HPMP 2. RPMP 3. Heating period 4. Tapping period). with an example of the electrical input. In this work the process phases are defined by the nature of the electrical input only, and do not describe the melting conditions inside the furnace. This definition is chosen because the role of the process periods in this work is to provide a reference for comparing different process phenomena.

The early stage of processing can be labelled a high power melting period (HPMP) since high arc power can be sustained due to the shielding effect of the scrap. When the scrap starts to melt, and a clear view between the arc and refractories is established, the arc power has to be reduced. Thus, this period can be labelled a reduced power melting period (RPMP). The late part of the EAF process can be referred to as a heating period since most of the scrap is already molten and the melt is being heated up to tapping temperature. The last part of the process is EAF tapping, in which the melt is poured out of the EAF.

\subsection{Alkali Emissions during Different Process Periods}

The most intense alkali emission peaks observed in the spectrum are related to the $\mathrm{Na}$ I $589 \mathrm{~nm}$ doublet, K I $766.490 \mathrm{~nm}$ and K I $769.896 \mathrm{~nm}$ transitions (Fig. 3). The intensities of the Na I $589 \mathrm{~nm}$ doublet and K I $769.896 \mathrm{~nm}$ emission lines follow similar trends in HPMP, as can be observed in Fig. 3. The consequence is that the intensity of alkali emission lines can be described with reasonable accuracy only with a single alkali emission line. A similar connection was also found in the all other EAF process periods. Due to this connection the analysis of the most intense alkali emission lines, $\mathrm{Na} \mathrm{I}$ and K I, mainly focuses on the $\mathrm{Na}$ I $589 \mathrm{~nm}$ doublet.

Average and standard deviations for the calculated $\mathrm{Na}$ I $589 \mathrm{~nm}$ peak areas, FWHMs and absorption coefficients during different process periods are presented in Table 2. It can be seen from Table 2 that the parameters of the $\mathrm{Na}$ I $589 \mathrm{~nm}$ emission line vary significantly between different process periods, which suggests that the conditions in which $\mathrm{Na}$ I optical emissions are generated differ in each process period. Examples of high area, low area and high absorption alkali peaks are presented in Fig. 4. The band structure in the $715 \mathrm{~nm}$ region is likely to be caused by the distortion of the optical system deviating from the defined correction values.

It is interesting to note that the intensity of the alkali emissions is highly correlated to the calculated spectrum temperature, which can be observed from Fig. 5 presenting the calculated temperatures as well as the $\mathrm{Na}$ I $589 \mathrm{~nm}$

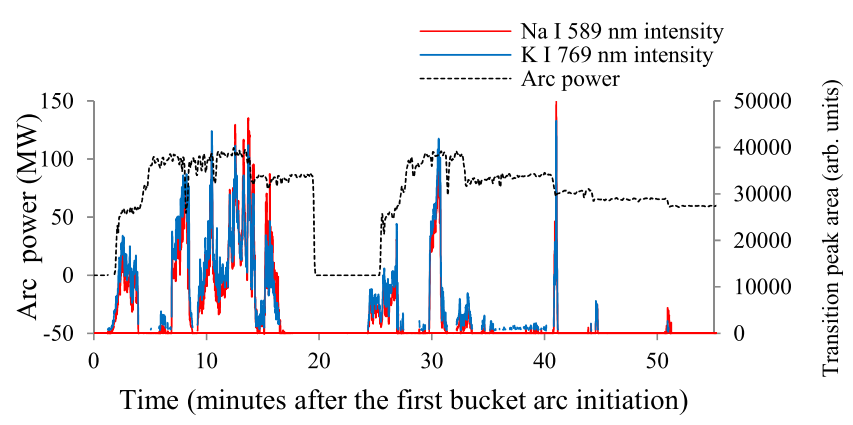

Fig. 3. Alkali emission lines during scrap melting. (Online version in color.) 
Table 2. Averages and standard deviations of $\mathrm{Na}$ indicators during different process periods.

\begin{tabular}{lllcccc}
\hline \multicolumn{1}{c}{ Indicator } & \multicolumn{1}{c}{ Unit } & & HPMP & RPMP & Heating & Tapping \\
\hline FWHM & $\mathrm{nm}$ & Average & 1.99 & 1.86 & 6.40 & 2.62 \\
& & Standard deviation & 1.12 & 1.03 & 3.02 & 1.79 \\
\multirow{2}{*}{ Absorption coefficient } & \multirow{2}{*}{ arb. units } & Average & 0.27 & 0.30 & 0.63 & 0.73 \\
& & Standard deviation & 0.15 & 0.27 & 0.48 & 0.61 \\
\multirow{3}{*}{ Area } & \multirow{3}{*}{ arb. units } & Average & 2370 & 6310 & 52200 & 8660 \\
& & Standard deviation & 2290 & 748 & 102000 & 43100 \\
\hline
\end{tabular}
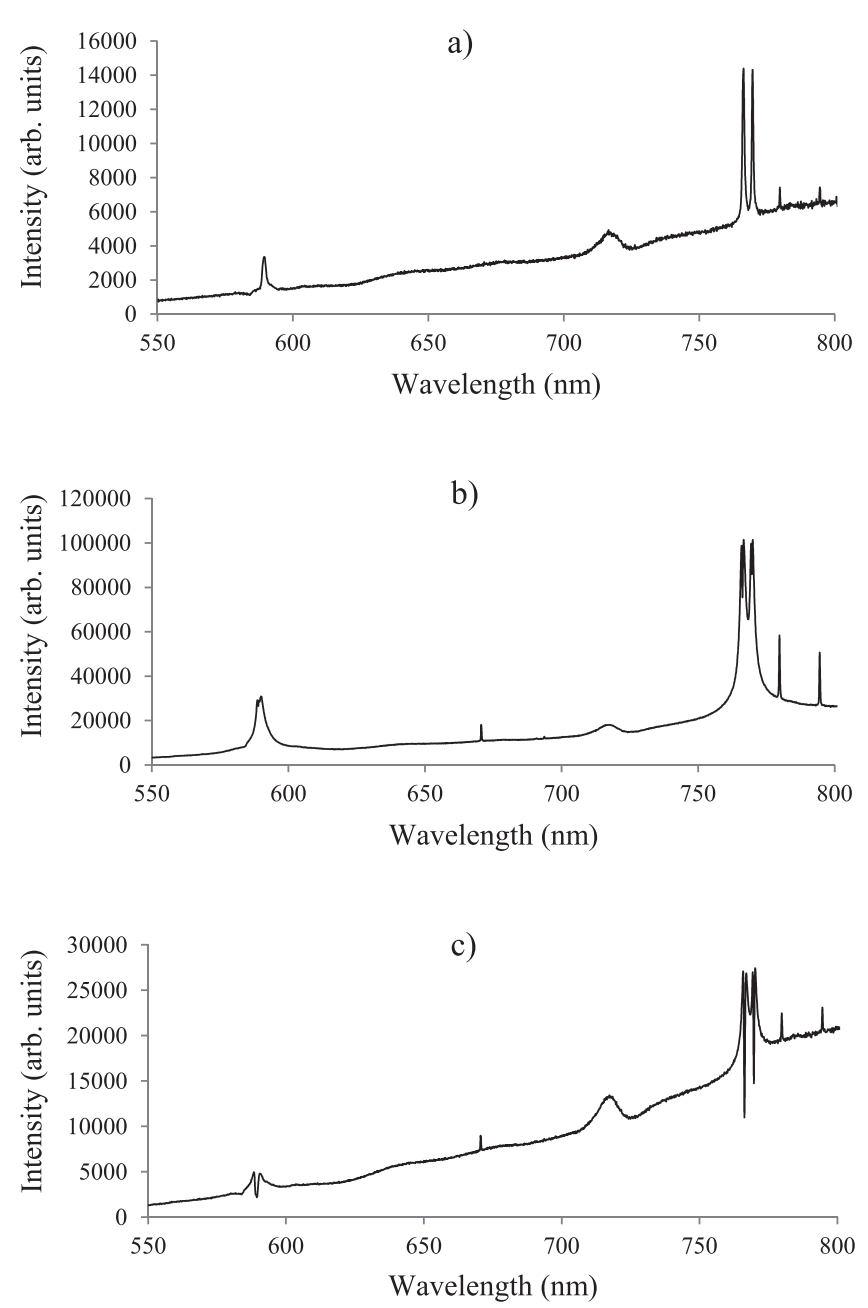

Fig. 4. Examples of different types of alkali emission spectra (a) small FWHM (b) high FWHM (c) absorption.

intensity. The correlation suggests that the alkali emissions are caused by the thermal decomposition of alkali oxides at high temperatures, which has been observed in pyrotechnic flames. ${ }^{12)}$ While the intensity of the alkali emission lines are highly dependent on the calculated spectrum temperature, the slope of the dependence was observed to vary between different scrap baskets. The likely explanation for this behavior is that the alkali emission intensity is also affected by the amount of alkali metals in the furnace.

\subsection{High Power Melting Period}

In a HPMP most important process phenomena are related to the behavior of the charge material. In the early stages of this period the dark current of the CCD and alkali

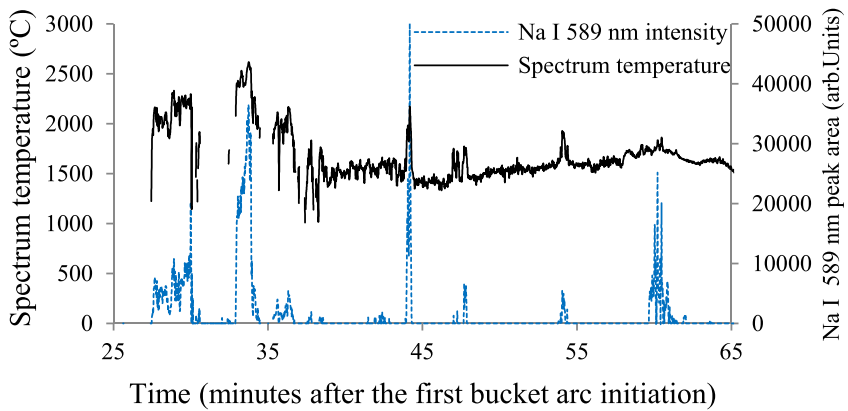

Fig. 5. An example of spectrum temperature and Na I $589 \mathrm{~nm}$ emission line intensity during a RPMP. (Online version in color.)

emission lines dominate the spectra. $37 \%$ of the total number of HPMP spectrum frames contain significant alkali emission lines. Alkali emission spectra and dark current dominated spectrum frames are observed subsequently, which means that the scrap in the view cone is still solid during this period.

\subsubsection{Occurrence of Alkali Emission Lines during HPMP}

The alkali emissions observed during the HPMP generally follow a similar pattern. Alkali emissions are observed a few minutes after the arc has been switched on and they are observed intermittently during the HPMP. When the scrap is nearly molten, the alkali emissions tend to decrease. The area of the alkali emission peaks vary, as can be seen from Fig. 3.

The occurrence of alkali peaks in the HPMP is likely to be related to the volatilization of organic components and moisture, as well as pure alkali components inside the scrap. This would explain why fewer alkali emission frames are observed towards the end of the HPMP.

The hypothesis that the source of alkali emissions being volatile components in the scrap was studied by comparing the area of the alkali emission lines to the off-gas temperature. It can be seen from Fig. 6 that the area of the Na I emission line has a clear relation to the off-gas temperature. The connection between off-gas temperature and the area of the $\mathrm{Na}$ I emission line suggests that the source of alkali emissions is the exothermic reactions in the furnace atmosphere, which can be caused by either volatilization of the scrap components or deoxidation reactions inside the steel melt.

In many cases the occurrence of alkali peaks is related to the material additions through the furnace roof. Figure 7 illustrates the connection between material charging and 


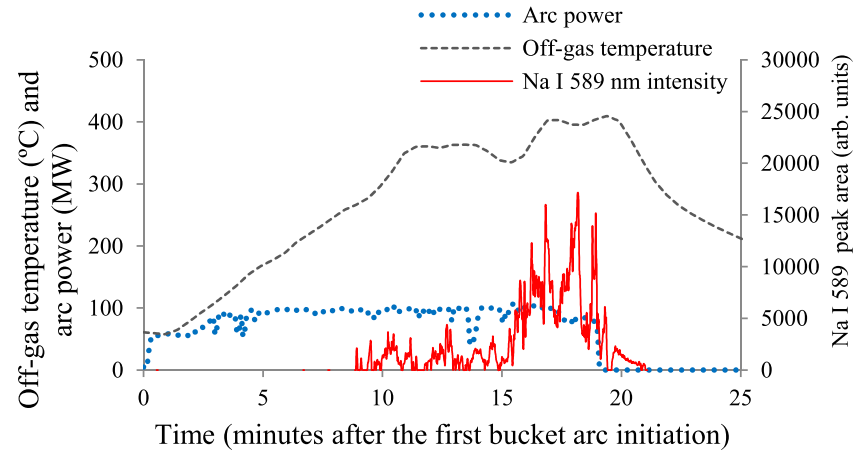

Fig. 6. The connection between EAF off-gas temperature and the area of the $\mathrm{Na}$ I $589 \mathrm{~nm}$ emission lines. (Online version in color.)

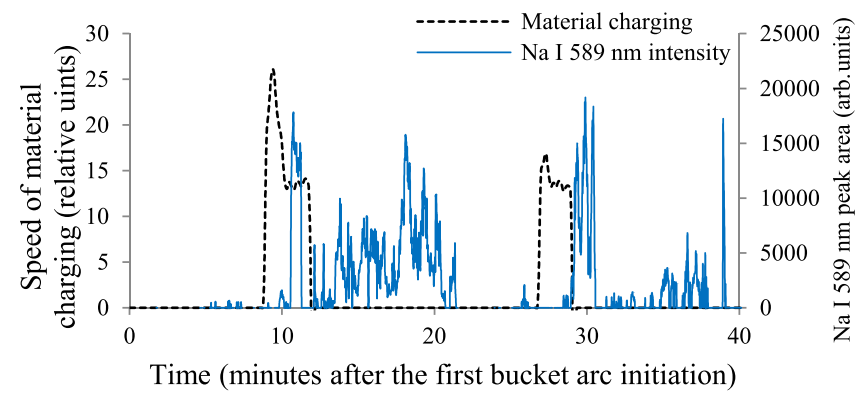

Fig. 7. The material charging and the area of the Na I $589 \mathrm{~nm}$ emission line. (Online version in color.)

alkali emission peaks. These alkali emissions are caused by exothermic gas phase reactions; material additions tend to generate flames visible even in the operator room. The temperatures calculated from the emission spectra associated with alkali peaks after material charging are typically over $2000^{\circ} \mathrm{C}$. This is not surprising considering the adiabatic flame temperature of the stoichiometric combustion of $\mathrm{CO}$ with $\mathrm{O}_{2}$ in a $78.1 \%$ nitrogen oxide atmosphere is $2592^{\circ} \mathrm{C} .{ }^{13)}$

Alkali emission lines during the HPMP have a low degree of absorption, as can be observed in Table 2. The low degree of absorption and relatively low intensity of alkali emission lines in melting periods suggest that gas generation leading to alkali transitions occur in relatively optically thin conditions. A low degree of absorption is surprising considering fuming has been observed to be most intense during these early periods. ${ }^{14)}$ However, a low degree of absorption can be explained by the small distance between the exothermic reactions and the optical fiber. During the melting periods the lower levels of furnace are full of scrap, which means that the alkali emission lines are generated in the upper parts of the furnace.

\subsection{Reduced Power Melting Period}

During the RPMP less alkali emission frames are observed and thermal radiation starts to dominate the emission spectra. Alkali peaks are only periodically observed during the RPMP and the rise in average emission intensity is attributed to an increase in the hot slag volume in the viewing cone. Significant alkali emission lines are observed in $8 \%$ of the total number of spectrum frames obtained during this period. This is illustrated in Fig. 8 where the connection between the average intensity and $\mathrm{Na}$ I $589 \mathrm{~nm}$ peak

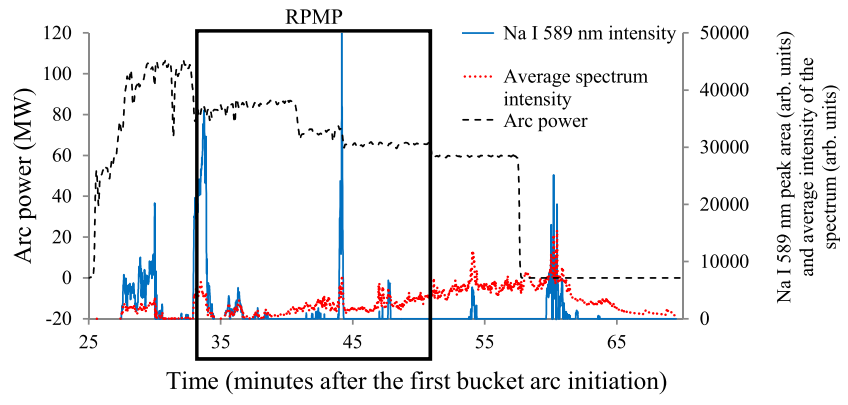

Fig. 8. A typical example of $\mathrm{Na}$ I $589 \mathrm{~nm}$ emission line intensity, average spectrum intensity and arc power during RPMP. (Online version in color.)

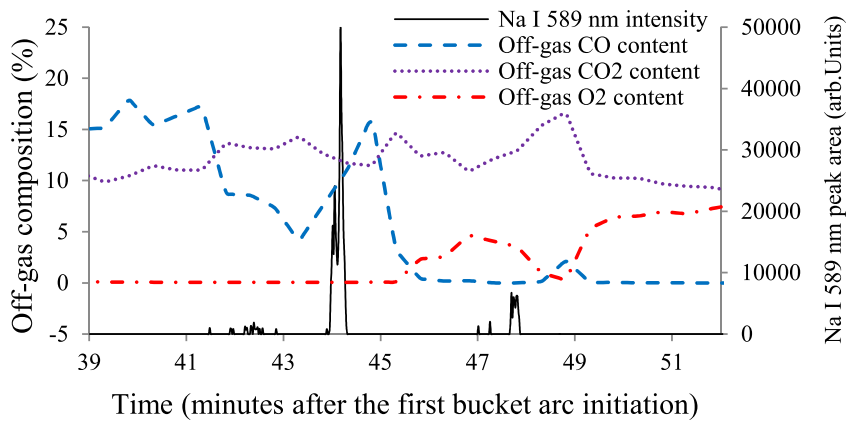

Fig. 9. The Na I $589 \mathrm{~nm}$ emission line intensity and off-gas composition. (Online version in color.)

intensity breaks during the RPMP. Also, during the early stages of this period spectrum frames are usually dominated by the dark current of the CCD, which means that very little light is emitted from the furnace. This is due to the depletion of the components causing exothermic reactions and the fiber seeing only solid scrap.

\subsubsection{Occurrence of Alkali Emission Lines during the RPMP}

During RPMP alkali emission lines are closely related to the sudden increase in off-gas $\mathrm{CO}$ content, as can be seen in Fig. 9. The dead time of the off-gas analysis is about 45 seconds, which causes a delay in the emission spectrum measurement. A likely explanation for these infrequent high off-gas CO peaks during the reduced melting period can be attributed to scrap collapsing during melting. The collapsing scrap causes solid scrap components to enter the melt, which can cause fast oxidation-deoxidation reactions. It is interesting to note that alkali emission lines are only related to the momentary high $\mathrm{CO}$ content of the off-gas. In Fig. 9 it can be observed that phenomena related to a constantly high $\mathrm{CO}$ content at the starting stage of the RPMP does not cause alkali transitions. This is probably due to the low amount of $\mathrm{O}_{2}$ in the furnace, which does not allow combustion of $\mathrm{CO}$ inside the furnace. On the other hand, the instantaneous increase in gas volume raises the furnace pressure and causes the gases to erupt from the furnace and come into contact with the oxygen in the ambient atmosphere.

\subsubsection{Analysis of Scrap Melting}

The change from a dark current dominated spectrum to a spectrum dominated by thermal radiation allows the analysis of scrap melting. Measuring this change will allow the 


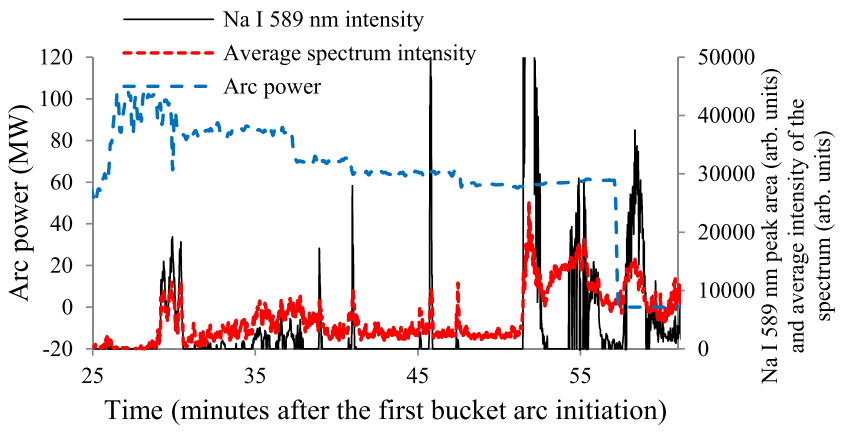

Fig. 10. The intensity of $\mathrm{Na}$ I $589 \mathrm{~nm}$ emission lines, the average spectrum intensity and the arc power during a heat with abundant alkali emissions in RPMP. (Online version in color.)

interpretation of scrap melting in EAFs. However, it is very important to differentiate the optically thin conditions from the optically thick. The melting behavior is not always as simple as seen in Fig. 8, heats can follow more confusing pattern as presented in Fig. 10.

When the exothermic reactions in the furnace atmosphere lead to optically thick atmosphere, the observed thermal radiation is mostly influenced by temperature of the off-gas and does not carry information on the scrap melting. Optically thick conditions are caused by increased dust generation associated with the bursting of $\mathrm{CO}$ bubbles on the slag surface. ${ }^{15)}$ These optically thick conditions must be identified when seeking accurate information on scrap melting for process control purposes.

To differentiate between optically thin and thick conditions, information about gas generation in the EAF is required. The gases generated inside the $\mathrm{EAF}(\mathrm{CO}$, volatile organic compounds) have a tendency to be highly reactive with oxygen, which provides a method for calculating an indication of the generation of these gases from the optical emission spectrum. The occurrence of these exothermic reactions can be observed from alkali emission lines as well as from high temperatures calculated from the thermal radiation curve. It can be seen from Fig. 5 that temperatures associated with high $\mathrm{Na}$ I 589 peak intensities are also high, generally over $1700^{\circ} \mathrm{C}$. This high temperature is likely generated by exothermic reactions in the gas phase, as discussed earlier.

The first criterion for scrap melting was defined as an average intensity of 60 frames becoming higher than 800 counts. This criterion describes the melting of the scrap in the viewing cone. The exact value of this criterion is arbitrary, and it was defined so that it represents the clearly molten conditions. Defining an exact number does not state precisely at what stage the scrap melting is, but provides a method for comparing the temporal differences in scrap melting of different heats.

The effect of alkali transitions was removed by setting a second criterion that the calculated temperature has to be below $1600^{\circ} \mathrm{C}$ in order to single out the optically thick conditions. Additionally, only the frames where the slope of the last 10 seconds was positive were deemed as being suitable scrap melting conditions. The negative temperature differential indicates cooling after exothermic reactions and in these cases the intensity is increased by these reactions

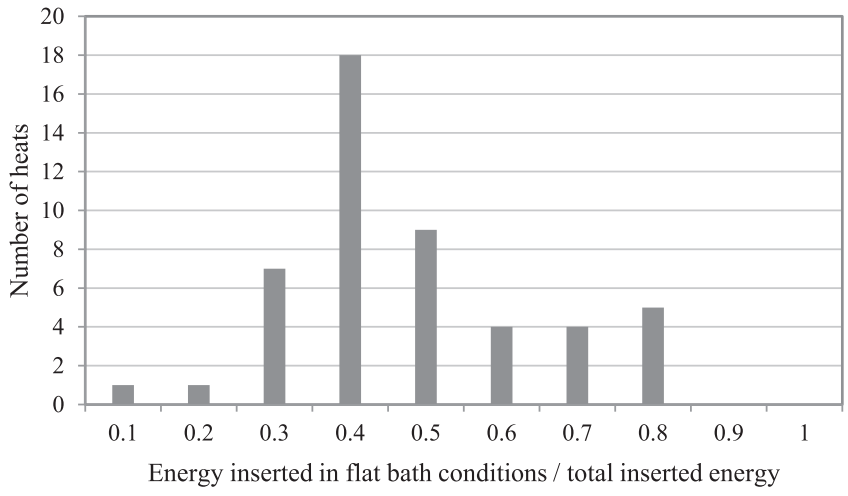

Fig. 11. Histogram of ratios between inserted energy in flat bath conditions and total inserted energy.

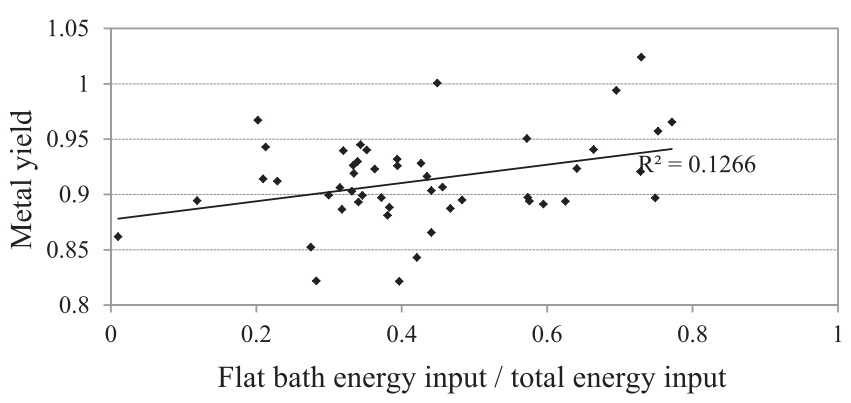

Fig. 12. Ratio of flat bath energy input to total energy inserted and metal yield.

and not the hot slag.

The first frame indicating scrap melting was used to analyze how much electrical energy was inserted before and after the scrap melting. The electrical input describes the melting conditions better than time since it is not susceptible to voltage ramp changes. The differing time of scrap melting with similar electrical inputs indicates differing scrap melting conditions.

The energy inserted after the scrap melting signal was found to vary significantly between the heats. It can be seen in Fig. 11 that in most of the heats the energy inserted after the scrap melting is between $30-50 \%$ from the total energy inserted. In some heats the scrap was found to melt much faster. The low number of heats with very late scrap melting is plausible since the viewing cone was aimed relatively close to the furnace center, in which the scrap melts earlier than near the furnace sides.

The validity of the scrap melting signal obtained from the emission spectra can be analyzed by comparing the differences in scrap melting times to differences between electrical and material properties of the heats. Certain caution with the results of the analysis should be employed since the overall heat properties are influenced by many other factors besides scrap melting, the most important of these being the scrap composition and the amount of material left from the previous heat on the bottom of the furnace.

Figure 12 shows a comparison between the lengths of the flat bath period and the overall metal yield. It can be seen from Fig. 12 that very low scrap yields of below 0.85 are not observed when more energy is inserted into the metal in flat bath conditions than in conditions where the scrap has not molten (a ratio over 0.5 ). The explanation for this 
is that when the scrap melts early enough, the whole scrap charge is molten by the end of the heat and possible accumulated material on the bottom of the furnace starts to melt. The connection between the overall metal yield of the heat and the scrap melting signal suggests that the scrap melting signal obtained from a single measurement cone describes the real melting conditions inside the furnace, although the correlation is not strong.

\subsection{Heating Period}

During the heating period thermal radiation is the dominating feature in the most of the measured spectra. Very intense alkali emission lines can also be occasionally observed. The most interesting phenomena in this period are related to the changes in measured spectrum temperature and the nature of the observed alkali emission lines. 10\% of the total number of emission spectra contains significant alkali emissions during the heating period.

\subsubsection{Alkali Emission Lines during the Heating Period}

During the heating period very intense and broad alkali emission lines are observed, as can be seen in Table 2 . These emission lines are attributed to fast and intense gas generation. The average $\mathrm{CO}$ content of the off-gas during intense gas generation is $5.2 \%$ during RPMP, while it is $9.5 \%$ in the heating period (below $1 \%$ off-gas content was filtered out from the calculation). The very intense gas generation in the heating period is surprising considering that the organic components and the moisture has already vaporized in this process period. The likely explanation for these alkali emission lines is poor mixing during the heating period. The reaction starts with the collapse of scrap or other process changes which introduce oxide material to the steel melt. Due to reduction of this oxide material (for example $\mathrm{FeO}$ ), a high amount of gas is generated locally. The increase in $\mathrm{CO}$ generation causes local foaming, which in turn increases mixing and introduces more oxide material to react with steel. The consequence is a reaction which spontaneously increases until somewhat more homogenous conditions are reached inside the furnace. The high amounts of instantaneous $\mathrm{CO}$ and $\mathrm{CO}_{2}$ measured in the off-gas presented in Fig. 8 suggest that the only plausible reason for intense alkali emission lines is an increase in gas generation.

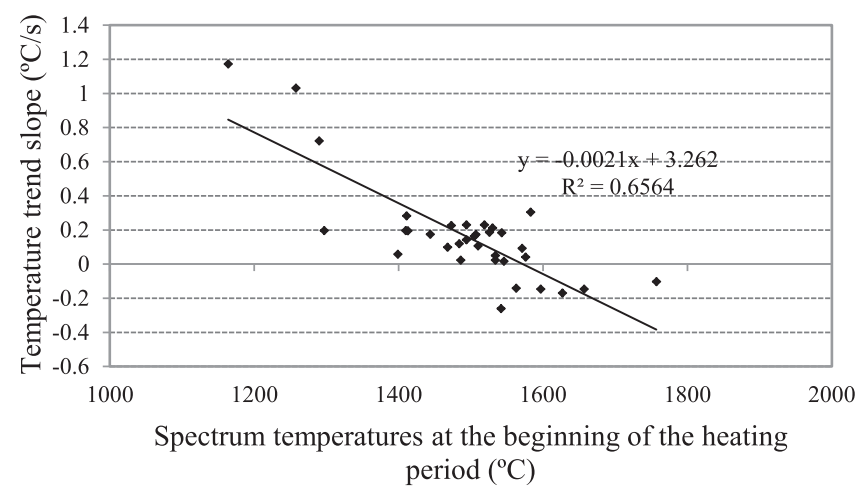

Fig. 13. The temperature trend and the temperatures at the beginning of the heating period.

\subsubsection{Temperature Evolution during the Heating Period}

Generally, there are three different types of temperature trends observed during the heating period. In 28 heats the temperature increases linearly during the heating period. In six heats the temperature decreases during the heating period and in the rest of the heats there is no clear temperature trend or the length of the heating period is not long enough to analyze long-term trends. Common for the linearly increasing temperature heats is the low starting temperature, whereas the heats having decreasing temperature trends tend to have a high starting temperature. The heats having no linear trends usually have high alkali emission lines during heating period. The speed of temperature change for different heats along with starting temperature is presented in Fig. 13.

The significant effect of the starting slag temperature on the temperature slope is natural because all the heats were heated with the same arc power during the heating period. Decreasing slag surface temperatures with high starting temperatures suggest that the electrical energy input during the heating period is lower than the thermal losses when the slag surface temperature is high enough. During the heating period the difference between the slag surface temperatures of the different heats decreases, but significant variation still exists. $60 \%$ of the heats had slag surface temperatures between $1550^{\circ} \mathrm{C}$ and $1650^{\circ} \mathrm{C}$ before tapping.

\subsubsection{Temperature Fluctuations during the Heating Period}

The temperature measured from the emission spectrum varies significantly even between subsequent spectrum frames, as can be seen from Fig. 14 showing an example of changes in the measured temperature during the heating period. This shows that the slag surface conditions are in a state of constant change.

In order to analyze the short-term fluctuations, long-term trends were removed from the data. First, the heats showing signs of non-linear temperature trends were filtered from the data to remove the effect of alkali transitions. Once the data contained only those heats with linear trends, the trends were approximated with linear functions and their respective equations were subtracted from the data. The result was data containing only short-term variations. The length and magnitude of these short-term variances were analyzed by calculating the average difference between the lagged time frames in the data. The average temperature differences between different time lags are presented in Fig. 15.

The average temperature difference between subsequent frames being $29^{\circ} \mathrm{C}$ shows that most of the fluctuations are

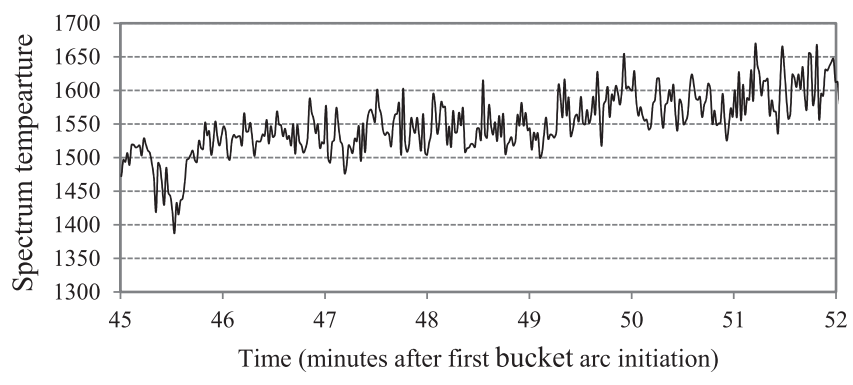

Fig. 14. An example of temperature evolution during a heating period. 


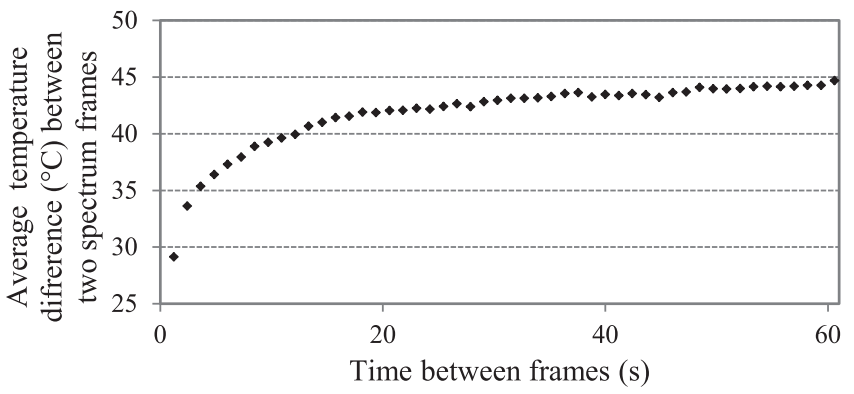

Fig. 15. Average temperature difference between two spectrum frames with increased lag values.

caused by phenomena lasting below the kinetic cycle of the measurement (1.2 seconds). However, the temperature difference increases rapidly when the lag is increased, which means that the measured temperatures have short-term trends. From the decreasing slope of the curve it can be seen that the almost maximum temperature difference is reached by a lag of 15 seconds. From this it can be surmised that the short-term trends in the data are below 15 seconds in length. The continuous slowly increasing period of standard deviation with long times between the frames can be interpreted as a residual of the removed long-term trends.

The possible nature of these short term fluctuations can be analyzed by their supposed duration. The phenomena lasting less than one second and causing most of the variation are likely related to the movement of the slag surface and fumes, as well as errors in temperature calculation caused by the fluctuation of the dark current in the CCD. Dark current is a temperature dependent phenomenon which causes a charge to appear in a CCD pixel without the collision of photons. ${ }^{16)}$ Short-term trends lasting below 15 seconds can be related to the macroscopic movement of the slag particles on the slag surface or changes in local gas generation and bubble bursting.

\subsection{Tapping Period}

Alkali emission lines and the temperature evolution during tapping depend very much on the heat in question and clear archetypes of heat are difficult to define. The emission spectrum is influenced by the generation $\mathrm{CO}$, which in turn depends on the heterogeneousness of the melt, which can vary significantly between heats. During the early part of the tapping spectrum, frames were found to be mostly dominated by alkali emission lines, and a total of $41 \%$ of the all spectrum frames obtained during the tapping period contain significant alkali emission lines. This is likely due to the tilting of the furnace, which causes the material accumulated at the sides of the furnace to collapse into the melt. The tilting of the furnace also increases mixing, but the increased mixing is not as intense as the mixing in the ladle. By the end of the tapping, alkali emission lines tend to decrease. Measured spectrum temperatures also vary between heats. Generally the trend is towards lower temperatures, since the slag surface cools during the tapping.

\subsubsection{Alkali Emission Lines during the Tapping Period}

The alkali emission lines during the tapping period differed from the alkali emission lines of other periods by their higher degree of absorption, as can be observed from
Table 2. The high degree of absorption suggests that the atmosphere between material emitting the alkali emission lines and the optical fiber contains high amounts of alkali metals in the tapping phase. The other explanation for high absorption is that the exothermic reaction emitting the alkali emission lines occurs farther away from the fiber than in earlier periods.

\section{Industrial Relevance}

The analysis of alkali emission lines and spectrum temperatures provided numerous insights into the exothermic reactions and slag surface conditions during different process stages. The most promising industrial applications for the new information are the analysis of local scrap melting and steel temperatures.

The occasional high optical thickness of EAF atmosphere caused by flames and fumes during HPMP and RPMP suggests that the optical emission spectrum data needs to be filtered when seeking information on slag or scrap surface conditions. With the aid of filtered emission spectrum data, it is possible to analyze the local scrap melting conditions in the EAF. This information is beneficial for EAF control because currently the methods for obtaining information on scrap melting are very limited. When scrap melting can be reliably measured, it will be possible to optimize the arc voltage steps and the use of burner fuels for the actual melting conditions inside the EAF. Optimization would allow better energy efficiency because the efficiency of the inserted electric energy drops once the scrap melts in the EAF.

During the heating period optical emission measurement allows the analysis of slag surface temperatures. The slag surface temperature can be used to validate various heat transfer models and possibly provide a rough estimate of steel temperatures. In the EAF of this study the surface temperatures did not correlate to the steel temperatures, but it could be possible with a furnace having sufficiently high mixing due to an electromagnetic stirrer (EMS) or bottom stirring argon. To verify the use of slag surface temperature in process control, the slag surface temperature from multiple measurement areas around the furnace should be measured.

\section{Conclusions}

This work describes the results of a test period conducted at Outokumpu Stainless Oy, Tornio Works, 140 t EAF. The focus of the study was the analysis of the spectrum types related to alkali emission lines and thermal radiation. The following conclusions can be drawn from the results:

(1) The alkali emission lines in high power melting period were found to be related to the exothermic reactions in the furnace atmosphere. The peak area of both $\mathrm{Na}$ I and $\mathrm{K}$ I alkali emission lines were found to change in a similar way, which suggests that the intensity of the alkali emission lines is related to the excitation conditions rather than the amount of alkali metals in the charge. The alkali emission lines during the high power melting period showed smaller signs of absorption than in other process stages.

(2) In the reduced power melting and heating period the 
alkali emission lines are periodic and related to the momentary high CO content of the off-gas. These alkali emission lines have very high peak area and are highly absorbed.

(3) Scrap melting during reduced power melting period was analyzed by calculating a scrap melting indicator from the increase in the average spectrum intensity. The calculated indicator has correlation with overall metal yield of the heat, which suggests that the calculated signal describes the melting in the EAF to a certain degree.

(4) In the heating period the alkali emission lines behave in a similar way as in the reduced power melting period, but the calculated surface temperature becomes more stable. 28 of the total 70 heats have clear linear trends in surface temperatures. The slope of the surface temperature trends depend on the surface temperature at the start of the heating period.

(5) The temperature difference between frames in the heating period indicates that short-term temperature trends in the heating period surface temperatures are less than 15 seconds in length.

(6) During the tapping period high numbers of spectrum frames dominated by alkali emission lines were observed. The alkali emission lines have a high degree of absorption during the tapping period.

\section{Acknowledgements}

The Finnish Funding agency for Technology and innovation (TEKES) and Technology Industries of Finland Centennial Foundation Fund for the Association of Finnish Steel and Metal Producers are acknowledged for funding of this work. Mr. Niko Hyttinen from Outokumpu Stainless Oy is acknowledged for overseeing the measurements in Tornio. Mr. Juho Kunelius, Mr. Kimmo Vallo and Dr. Paavo Hooli from Outokumpu Stainless Oy are acknowledged for helping to arrange the measurements and providing the reference data.

\section{REFERENCES}

1) H. D. Goodfellow, L. Ferro and P. Galbiati: Ironmaking Steelmaking, 32 (2005), 216.

2) G. R. Peacock: Proc. SPIE 4020: Thermosense XXII, SPIE, Bellingham, (2000).

3) T. Jansen, K. Krüger, H. Schliephake, B. Dettmer and L. Schlinge: Proc. 10th EEC, ASMET, Leoben, (2012).

4) Q. Wu, M. J. Thomson and A. Chanda: Metall. Mater. Trans. B, 36B (2005), 53.

5) R. Kühn, H. G. Geck and K. Schwerdtfeger: ISIJ Int., 45 (2005), 1587.

6) L. Lei and M. Zhizhong: Neurocomputing, 82 (2012), 91.

7) A. Yazdani, M. L. Crow and J. Guo: IEEE Trans. Power Del., 24 (2009), 2284.

8) M. Aula, A. Leppänen, J. Roininen, E.-P. Heikkinen, K. Vallo, T. Fabritius and M. Huttula: Metall. Mater. Trans. B, 45B (2014), 839.

9) S. Rego-Barcena, R. Mani, F. Yang, R. Saari and M. J. Thomson: Metall. Mater. Trans. B, 40B (2009), 158.

10) Anonymous: Teräskirja, Metallinjalostajat ry, Helsinki, Tampere (2009), 104.

11) R. Brun and F. Rademakers: Nucl. Inst. \& Meth. in Phys. Res. A, 389 (1997), 81

12) D. R. Dillehay: Resonance Line Broadening of Alkali Metals in Pyrotechnic Flames, PN, Longhorn Army Ammunation Plant, Texas, (1983).

13) A. Roine: HSC Chemistry 7, Outotec, Espoo, (2009).

14) B. Bowman and K. Krüger: Arc Furnace Physics, Verlag Stahleisen GmbH, Düsseldorf, (2009), 247.

15) A. G. Guézennec, J. C. Huber, F. Patisson, P. Sessiecq, J. P. Birat and D. Ablitzer: Powder Technol., 157 (2005), 2.

16) R. L. Baer: Proc. SPIE 6068: Sensors, Cameras, and Systems for Scientific/Industrial Applications VI, SPIE, Bellingham, (2006). 